\title{
Reproducibility of intravascular ultrasound radiofrequency data analysis: implications for the design of longitudinal studies
}

\author{
Gastón A. Rodriguez-Granillo ${ }^{1}$, Sophia Vaina ${ }^{1}$, Héctor M. García-García ${ }^{1}$, \\ Marco Valgimigli ${ }^{1}$, Eric Duckers ${ }^{1}$, Robert J. van Geuns ${ }^{1}$, Evelyn Regar ${ }^{1}$, \\ William J. van der Giessen ${ }^{1}$, Marco Bressers ${ }^{2}$, Dick Goedhart ${ }^{2}$, Marie-Angele Morel $^{2}$, \\ Pim J. de Feyter ${ }^{1}$ \& Patrick W. Serruys ${ }^{1}$ \\ ${ }^{1}$ Department of Interventional Cardiology of the Erasmus Medical Center, Rotterdam, The Netherlands; \\ ${ }^{2}$ Cardialysis $B V$, Rotterdam, The Netherlands
}

Received 24 November 2005; accepted in revised form 19 January 2006

Key words: agreement, atherosclerosis, plaque characterization, reproducibility, ultrasonography

\begin{abstract}
Objectives: The purpose of this study was to assess in vivo the reproducibility of tissue characterization using spectral analysis of intravascular ultrasound (IVUS) radiofrequency data (IVUS-VH). Background: Despite the need for reproducibility data to design longitudinal studies, such information remains unexplored. Methods and results: IVUS-VH (Volcano Corp., Rancho Cordova, USA) was performed in patients referred for elective percutaneous intervention and in whom a non-intervened vessel was judged suitable for a safe IVUS interrogation. The IVUS catheters used were commercially available catheters (20 MHz, Volcano Corp., Rancho Cordova, USA). Following IVUS-VH acquisition, and after the disengagement and re-engagement of the guiding catheter, an additional acquisition was performed using a new IVUS catheter. Fifteen patients with 16 non-significant lesions were assessed by 2 independent observers. The relative inter-catheter differences regarding geometrical measurements were negligible for both observers. The inter-catheter relative difference in plaque cross-sectional area (CSA) was $3.2 \%$ for observer 1 and $0.5 \%$ for observer 2 . The limits of agreement for (observer 1 measurements) lumen, vessel, plaque and plaque burden measurements were $0.82,-1.10 \mathrm{~mm}^{2} ; 0.80,-0.66 \mathrm{~mm}^{2} ; 1.08,-0.66 \mathrm{~mm}^{2}$; and $5.83,-3.89 \%$; respectively. Limits of agreement for calcium, fibrous, fibrolipidic and necrotic core CSA measurements were $0.22,-0.25 \mathrm{~mm}^{2} ; 1.02,-0.71 \mathrm{~mm}^{2} ; 0.61,-0.65 \mathrm{~mm}^{2}$; and $0.43,-0.38 \mathrm{~mm}^{2}$ respectively. Regarding the inter-observer agreement, the limits of agreement for lumen, vessel, plaque and plaque burden measurements were $2.61,-2.09 \mathrm{~mm}^{2} ; 2.20-3.03 \mathrm{~mm}^{2} ; 1.70,-3.04 \mathrm{~mm}^{2}$; and $9.16,-16.41 \%$; respectively, and for calcium, fibrous, fibrolipidic and necrotic core measurements of $0.08,-0.09 \mathrm{~mm}^{2} ; 0.89$, $-1.28 \mathrm{~mm}^{2} ; 0.74,-1.06 \mathrm{~mm}^{2}$; and $0.16,-0.20 \mathrm{~mm}^{2}$; respectively. Conclusions: The present study demonstrates that the geometrical and compositional output of IVUS-VH is acceptably reproducible.
\end{abstract}

\section{Introduction}

Intravascular ultrasound (IVUS) imaging has been shown to provide safe, accurate, real-time, tomographic measurements of coronary vessels in vivo
[1-4]. Over the past decade, IVUS has been used to describe the extent, severity, distribution, and morphology of coronary atherosclerosis [5-7]. Furthermore, several studies have evaluated the temporal effect of conventional and novel medical 
therapies on plaque progression by means of IVUS [8-10].

Since the fate of coronary atherosclerotic plaques has been related to their histological composition [11], precise in-vivo tissue characterization could provide important additional information and become a target for future drug therapy studies.

In-vitro studies have shown that visual interpretation of IVUS gray-scale images for plaque characterization is imprecise, in particular when assessing heterogeneous, lipid-rich plaques [12]. This has lead investigators to explore the radiofrequency data analysis, a potential source for in-vivo tissue characterization. Indeed, a recent ex-vivo study on explanted coronary segments showed that plaque characterization using spectral analysis of IVUS radiofrequency data (IVUS-VH) was feasible and provided a high predictive accuracy to estimate the composition of atherosclerotic plaques [13]. Several in-vivo studies have been conducted thereafter using this approach [14-17]. Nevertheless, although prior knowledge about the reproducibility of measurements are essential for the internal validity of any study using this technique, to date, only indirect evidence on the reproducibility of the technique is available [18]. Accordingly, we sought to study the inter-observer and inter-catheter agreement of IVUS-VH measurements at a single time-point.

\section{Methods}

\section{Patient population}

This was a single-center prospective, investigatorsdriven study that sought to explore in vivo the reproducibility of spectral analysis of IVUS radiofrequency data (IVUS-VH, Volcano Corp., Rancho Cordova, USA). The study population consisted of consecutive patients that were referred for elective percutaneous intervention and in whom a non-intervened vessel was judged suitable for a safe IVUS interrogation of a vessel segment of at least $30 \mathrm{~mm}$.

Exclusion criteria included the presence of severe calcification, vessel tortuosity, and haemodynamic instability. The study protocol was approved by the institutional ethics committee and a written informed consent was obtained from all patients.

\section{IVUS-VH}

IVUS-VH evaluates different spectral parameters of the radiofrequency data ( $\mathrm{Y}$-intercept, minimum power, maximum power, mid-band power, frequency at minimum power, frequency at maximum power, slope, etc.) to construct tissue maps that classify plaque into four major components. In preliminary in vitro studies, four histological plaque components (fibrous, fibrolipidic, necrotic core and calcium) were correlated with a specific spectrum of the radiofrequency signal [13]. These different plaque components were assigned color codes. Calcified, fibrous, fibrolipidic and necrotic core regions were labeled white, green, greenishyellow and red respectively.

\section{IVUS-VH acquisition}

The IVUS catheters used were commercially available phased array catheters (Eagle Eye Gold $^{\mathrm{TM}} 2.9$ F $20 \mathrm{MHz}$, Volcano Corp., Rancho Cordova, USA). The catheter probe was advanced at least $10 \mathrm{~mm}$ distal to a clearly visible side-branch and angiographic cine runs, before and during contrast injection, were performed to define the position of the IVUS catheter before the pullback was started. Using an automated pullback device, the transducer was withdrawn at a continuous speed of $0.5 \mathrm{~mm} / \mathrm{s}$. IVUS-VH acquisition was ECG-gated and acquired using a dedicated console (Volcano Corporation, Rancho Cordova, USA). IVUS-VH data was acquired after intra-coronary administration of isosorbide dinitrate and data was stored on DVD. Subsequently, and after the disengagement and re-engagement of the guiding catheter, the same procedure was performed using a new catheter (Eagle Eye Gold ${ }^{\mathrm{TM}} 2.9 \mathrm{~F} 20 \mathrm{MHz}$, Volcano Corp., Rancho Cordova, USA) and with the same side-branches as landmarks.

\section{IVUS-VH analysis}

IVUS-VH analysis was performed by an independent core laboratory (Cardialysis BV, Rotterdam, 
The Netherlands) using a semi-automatic contour detection software (IVUSLab 4.4, Volcano Corp., Rancho Cordova, USA). A region of interest (ROI) was identified using the inner side of two clear side-branches as reference and avoiding the presence of large side-branches within the ROI. Subsequently, the same ROI was identified in the other catheter's acquisition using side-by-side longitudinal and cross-sectional, contour-free views.

Contour detection of the lumen and the mediaadventitia interface was performed by 2 independent experienced IVUS analysts. The same 2 IVUS analysts re-analyzed the same cases, leading to the possibility of multiple comparisons: 2 sets (observers 1 and 2) of intra-catheter agreement, and 1 set of inter-observer agreement [observer 1 (catheters 1 and 2) vs. observer 2 (catheters 1 and 2)].

The contours of the external elastic membrane (EEM) and the lumen-intima interface enclosed an area that was defined as the coronary plaque plus media area. Geometrical and compositional data were obtained for each cross-sectional area (CSA) and an average was calculated for each ROI. Plaque burden was calculated as $\left[\left(\mathrm{EEM}_{\text {area }}\right.\right.$ - Lumen $\left.\left._{\text {area }} / \mathrm{EEM}_{\text {area }}\right) \times 100\right]$. The lumen and vessel eccentricity indexes were calculated dividing the minimum (lumen and vessel, respectively) diameter by the maximum diameter, whereas the plaque eccentricity index was calculated dividing the minimum plaque thickness by the maximum plaque thickness.

\section{Statistical analysis}

Discrete variables are presented as counts and percentages. Continuous variables are presented as means $\pm \mathrm{SD}$. The inter-observer and inter-catheter agreement were assessed using Bland-Altman plots [19]. This method plots the mean against the difference in measurements. Limits of agreement were determined by adding two standard deviations to the mean difference for the upper limit and by substracting two standard deviations from the mean difference for the lower limit. A two-sided $p$ value of less than 0.05 indicated statistical significance.

\section{Results}

Fifteen consecutive patients with 16 non-significant lesions were included in the study. Baseline characteristics of the patients included are depicted in Table 1. The study vessel was the left anterior descending in 9 patients $(60.0 \%)$, the left circumflex in $5(33.3 \%)$, and the right coronary artery in 1 patient $(6.7 \%)$. There were no peri-procedural complications.

\section{Inter-catheter agreement}

The studied length determined by landmarks was $19.71 \pm 10.5 \mathrm{~mm}$ for catheter 1 and $21.01 \pm 11.1$ $\mathrm{mm}$ for catheter $2(p=0.32)$. Geometrical and compositional data of matched ROIs interrogated with IVUS-VH using 2 subsequent $20 \mathrm{MHz}$ catheters are extensively depicted in Tables 2 and 3. The relative inter-catheter differences regarding geometrical measurements were negligible for both observers. Of note, the inter-catheter relative difference in plaque CSA was $3.2 \%$ for observer 1 and $0.5 \%$ for observer 2 . Only other less common indirect measurements such as plaque eccentricity and plaque minimal thickness showed relative differences $>5 \%$. Compositional measurements showed higher relative differences, although not

Table 1. Study population $(n=15)$.

\begin{tabular}{lc}
\hline & $n(\%)$ \\
\hline Age (years \pm SD) & $63.1 \pm 8.6$ \\
Male sex & $8(53.3)$ \\
Diabetes & $2(13.3)$ \\
Hypertension & $11(73.3)$ \\
Current smoking & $1(6.7)$ \\
Previous smoking & $5(33.3)$ \\
Hypercholesterolemia & $8(53.3)$ \\
Family history of coronary disease & $9(60.0)$ \\
Lipid lowering agents & $11(73.3)$ \\
Clinical presentation & \\
$\quad$ Stable angina & $14(93.3)$ \\
$\quad$ Unstable angina & $1(6.7)$ \\
Study vessel & \\
$\quad$ Left anterior descending & $9(60.0)$ \\
$\quad$ Left circumflex & $5(33.3)$ \\
$\quad$ Right coronary artery & $1(6.7)$ \\
\hline
\end{tabular}


Table 2. Mean CSA geometrical measurements of matched ROI with two subsequent $20 \mathrm{MHz}$ IVUS imaging catheters ( $n: 16)$.

\begin{tabular}{|c|c|c|c|c|}
\hline & Catheter 1 & Catheter 2 & Absolute $\Delta$ & Relative $\Delta(\%)$ \\
\hline \multicolumn{5}{|l|}{ Observer 1} \\
\hline Lumen CSA $\left(\mathrm{mm}^{2}\right)$ & $11.08 \pm 3.5$ & $10.94 \pm 3.5$ & $0.14 \pm 0.5$ & 1.3 \\
\hline Lumen max. diameter (mm) & $4.03 \pm 0.7$ & $4.01 \pm 0.6$ & $0.02 \pm 0.1$ & 0.4 \\
\hline Lumen min. diameter (mm) & $3.37 \pm 0.6$ & $3.34 \pm 0.6$ & $0.03 \pm 0.1$ & 0.9 \\
\hline Lumen mean diameter (mm) & $3.69 \pm 0.6$ & $3.67 \pm 0.6$ & $0.02 \pm 0.1$ & 0.6 \\
\hline Lumen eccentricity & $0.84 \pm 0.0$ & $0.83 \pm 0.0$ & $0.00 \pm 0.0$ & 0.5 \\
\hline Vessel CSA $\left(\mathrm{mm}^{2}\right)$ & $17.40 \pm 4.0$ & $17.46 \pm 4.0$ & $0.07 \pm 0.4$ & 0.4 \\
\hline Vessel max. diameter (mm) & $4.92 \pm 0.6$ & $4.93 \pm 0.6$ & $0.01 \pm 0.1$ & 0.3 \\
\hline Vessel min. diameter (mm) & $4.36 \pm 0.6$ & $4.37 \pm 0.6$ & $0.01 \pm 0.1$ & 0.2 \\
\hline Vessel mean diameter $(\mathrm{mm})$ & $4.63 \pm 0.6$ & $4.64 \pm 0.6$ & $0.01 \pm 0.0$ & 0.2 \\
\hline Vessel eccentricity & $0.89 \pm 0.0$ & $0.89 \pm 0.0$ & $0.00 \pm 0.0$ & 0.1 \\
\hline Plaque CSA $\left(\mathrm{mm}^{2}\right)$ & $6.32 \pm 2.0$ & $6.53 \pm 2.1$ & $0.21 \pm 0.4$ & 3.2 \\
\hline Plaque max. thickness (mm) & $1.01 \pm 0.2$ & $1.02 \pm 0.2$ & $0.01 \pm 0.1$ & 0.7 \\
\hline Plaque min. thickness (mm) & $0.09 \pm 0.1$ & $0.09 \pm 0.1$ & $0.01 \pm 0.0$ & 8.2 \\
\hline Plaque eccentricity $(\mathrm{mm})$ & $0.09 \pm 0.1$ & $0.10 \pm 0.1$ & $0.01 \pm 0.0$ & 10.0 \\
\hline Plaque burden $(\%)$ & $36.80 \pm 9.9$ & $37.77 \pm 9.9$ & $0.97 \pm 2.4$ & 2.6 \\
\hline \multicolumn{5}{|l|}{ Observer 2} \\
\hline Lumen CSA $\left(\mathrm{mm}^{2}\right)$ & $10.66 \pm 3.8$ & $10.67 \pm 3.8$ & $0.01 \pm 0.4$ & 0.1 \\
\hline Lumen max. diameter (mm) & $3.90 \pm 0.7$ & $3.91 \pm 0.7$ & $0.01 \pm 0.1$ & 0.2 \\
\hline Lumen min. diameter $(\mathrm{mm})$ & $3.34 \pm 0.6$ & $3.33 \pm 0.6$ & $0.01 \pm 0.1$ & 0.3 \\
\hline Lumen mean diameter (mm) & $3.61 \pm 0.6$ & $3.61 \pm 0.7$ & $0.00 \pm 0.1$ & 0.0 \\
\hline Lumen eccentricity & $0.86 \pm 0.0$ & $0.85 \pm 0.0$ & $0.01 \pm 0.0$ & 0.6 \\
\hline Vessel CSA $\left(\mathrm{mm}^{2}\right)$ & $17.76 \pm 4.0$ & $17.80 \pm 4.0$ & $0.05 \pm 0.4$ & 0.3 \\
\hline Vessel max. diameter (mm) & $4.95 \pm 0.6$ & $4.96 \pm 0.6$ & $0.01 \pm 0.1$ & 0.3 \\
\hline Vessel min. diameter (mm) & $4.41 \pm 0.6$ & $4.42 \pm 0.6$ & $0.01 \pm 0.1$ & 0.1 \\
\hline Vessel mean diameter (mm) & $4.68 \pm 0.6$ & $4.69 \pm 0.6$ & $0.01 \pm 0.0$ & 0.1 \\
\hline Vessel eccentricity & $0.89 \pm 0.0$ & $0.89 \pm 0.0$ & $0.00 \pm 0.0$ & 0.1 \\
\hline Plaque CSA $\left(\mathrm{mm}^{2}\right)$ & $7.10 \pm 2.1$ & $7.13 \pm 2.2$ & $0.03 \pm 0.4$ & 0.5 \\
\hline Plaque max. thickness (mm) & $1.05 \pm 0.2$ & $1.04 \pm 0.3$ & $0.00 \pm 0.1$ & 0.2 \\
\hline Plaque min. thickness (mm) & $0.16 \pm 0.1$ & $0.16 \pm 0.1$ & $0.01 \pm 0.0$ & 4.7 \\
\hline Plaque eccentricity (mm) & $0.16 \pm 0.1$ & $0.17 \pm 0.1$ & $0.01 \pm 0.0$ & 6.7 \\
\hline Plaque burden $(\%)$ & $40.75 \pm 10.7$ & $40.93 \pm 11.2$ & $0.19 \pm 1.8$ & 0.5 \\
\hline
\end{tabular}

LCSA, VCSA and PCSA refer to lumen, vessel and plaque CSAs. Plaque burden was calculated as $\left[\left(\mathrm{EEM}_{\text {area }}-\mathrm{Lumen}_{\text {area }} /\right.\right.$ $\left.\left.\mathrm{EEM}_{\text {area }}\right) \times 100\right]$.

exceeding $10 \%$, except from calcium $(11 \%)$ for observer 1 and fibrolipidic tissue $(13 \%)$ for observer 2 (Table 3). Indeed, Bland-Altman plots showed a good inter-catheter agreement for geometrical (Figure 1) and compositional (Figure 2) measurements. The limits of agreement for (observer 1 measurements) lumen, vessel, plaque and plaque burden measurements were 0.82 , $-1.10 \mathrm{~mm}^{2} ; 0.80,-0.66 \mathrm{~mm}^{2} ; 1.08,-0.66 \mathrm{~mm}^{2}$; and $5.83,-3.89 \%$; respectively. Limits of agreement for calcium, fibrous, fibrolipidic and necrotic core CSA measurements were $0.22,-0.25 \mathrm{~mm}^{2}$; $1.02,-0.71 \mathrm{~mm}^{2} ; 0.61,-0.65 \mathrm{~mm}^{2}$; and 0.43 , $-0.38 \mathrm{~mm}^{2}$ respectively.

\section{Inter-observer agreement}

For the assessment of the inter-observer agreement, a comparison between the same matched cross-sections (653 frames for catheter 1 and 663 frames for catheter 2) were analyzed by 2 independent observers. These 2 datasets were merged resulting in a paired inter-observer agreement evaluation of 1316 frames.

Inter-observer differences were larger than the inter-catheter measurements (performed by the same observer). This was particularly noticed in indirect measurements such as plaque CSA (10\%), plaque minimal thickness $(53 \%)$ and plaque 
Table 3. Mean CSA compositional measurements of matched ROI with two subsequent $20 \mathrm{MHz}$ IVUS imaging catheters ( $n$ :16).

\begin{tabular}{lcccc}
\hline & Catheter 1 & Catheter 2 & Absolute $\Delta$ & Relative $\Delta(\%)$ \\
\hline Observer 1 & & & & \\
Calcium CSA $\left(\mathrm{mm}^{2}\right)$ & $0.17 \pm 0.3$ & $0.16 \pm 0.2$ & $0.01 \pm 0.1$ & 8.0 \\
Calcium (\%) & $4.27 \pm 5.2$ & $3.82 \pm 3.8$ & $0.45 \pm 2.6$ & 11.1 \\
Fibrous CSA $\left(\mathrm{mm}^{2}\right)$ & $1.96 \pm 1.0$ & $2.11 \pm 1.1$ & $0.16 \pm 0.4$ & 7.7 \\
Fibrous $(\%)$ & $60.37 \pm 9.2$ & $62.15 \pm 8.7$ & $1.78 \pm 10.0$ & 2.9 \\
Fibrolipidic CSA $\left(\mathrm{mm}^{2}\right)$ & $0.66 \pm 0.4$ & $0.63 \pm 0.3$ & $0.02 \pm 0.3$ & 3.5 \\
Fibrolipidic $(\%)$ & $21.10 \pm 9.8$ & $19.58 \pm 7.0$ & $1.53 \pm 8.3$ & 7.5 \\
Necrotic core CSA $\left(\mathrm{mm}^{2}\right)$ & $0.40 \pm 0.4$ & $0.43 \pm 0.4$ & $0.02 \pm 0.2$ & 5.7 \\
Necrotic core $(\%)$ & $11.27 \pm 6.8$ & $10.87 \pm 6.6$ & $0.40 \pm 5.4$ & 3.6 \\
Observer 2 & & & \\
Calcium CSA $\left(\mathrm{mm}^{2}\right)$ & $0.17 \pm 0.3$ & $0.16 \pm 0.2$ & $0.01 \pm 0.1$ & 8.7 \\
Calcium $(\%)$ & $4.08 \pm 5.0$ & $3.74 \pm 3.4$ & $0.34 \pm 2.4$ & 8.7 \\
Fibrous CSA $\left(\mathrm{mm}^{2}\right)$ & $2.21 \pm 1.1$ & $2.28 \pm 1.2$ & $0.07 \pm 0.4$ & 3.1 \\
Fibrous $(\%)$ & $58.12 \pm 10.5$ & $60.63 \pm 7.9$ & $2.51 \pm 10.1$ & 4.2 \\
Fibrolipidic CSA $\left(\mathrm{mm}^{2}\right)$ & $0.88 \pm 0.6$ & $0.77 \pm 0.4$ & $0.11 \pm 0.4$ & 13.1 \\
Fibrolipidic $(\%)$ & $22.97 \pm 11.7$ & $20.95 \pm 10.8$ & $2.01 \pm 9.6$ & 9.2 \\
Necrotic core CSA $\left(\mathrm{mm}^{2}\right)$ & $0.42 \pm 0.4$ & $0.45 \pm 0.5$ & $0.03 \pm 0.2$ & 6.1 \\
Necrotic core $(\%)$ & $10.75 \pm 6.9$ & $11.02 \pm 6.5$ & $0.26 \pm 5.5$ & 2.4 \\
\hline
\end{tabular}

LCSA, VCSA and PCSA refer to lumen, vessel and plaque CSAs. Plaque burden was calculated as $\left[\left(\mathrm{EEM}_{\text {area }}-\mathrm{Lumen}_{\text {area }} /\right.\right.$ $\left.\left.\mathrm{EEM}_{\text {area }}\right) \times 100\right]$.

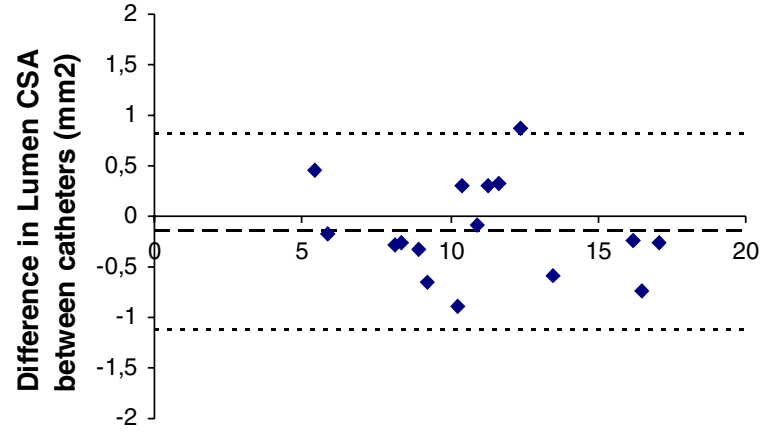

Mean Lumen CSA (mm2)

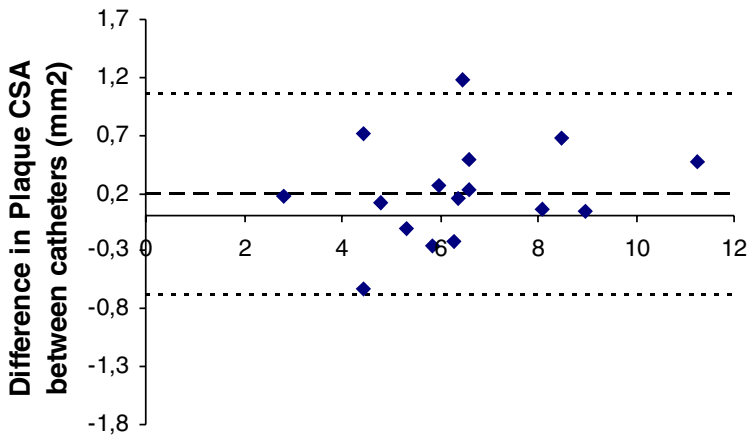

Mean Plaque CSA (mm2)

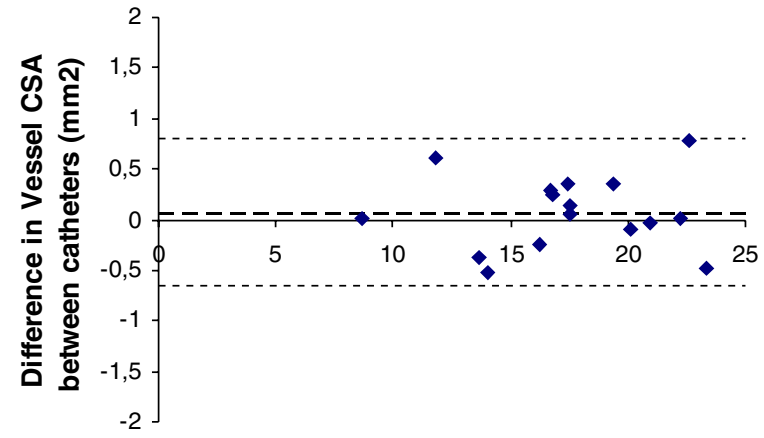

Mean Vessel CSA (mm2)

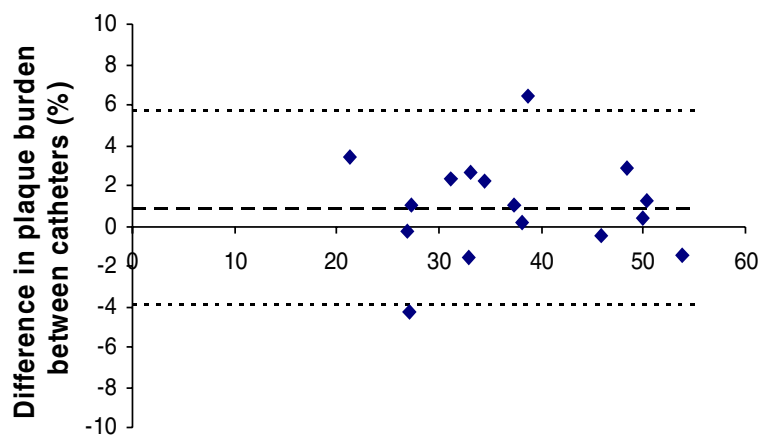

Mean Plaque burden (\%)

Figure 1. Bland-Altman plots depicting the (observer 1) agreement between catheters for geometrical measurements $(n=16)$. 


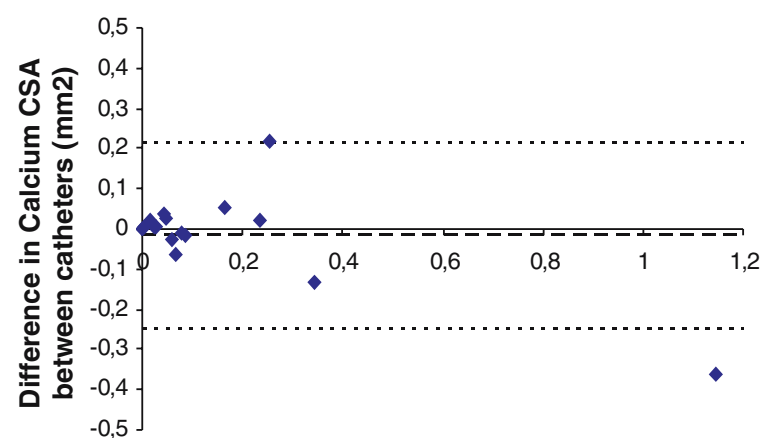

Mean Calcium CSA (mm2)

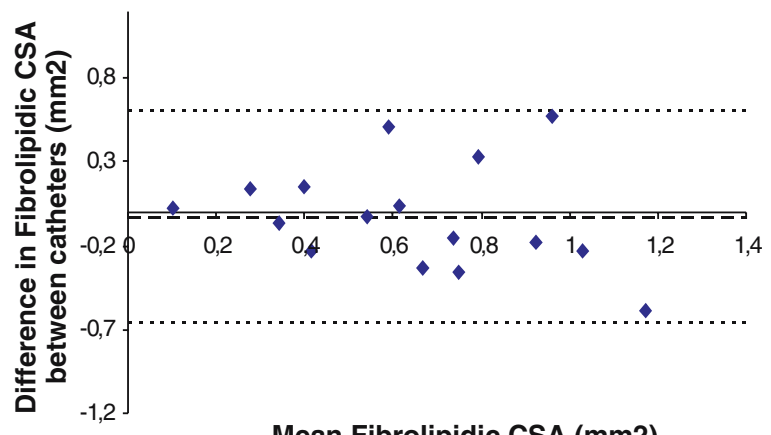

Mean Fibrolipidic CSA (mm2)

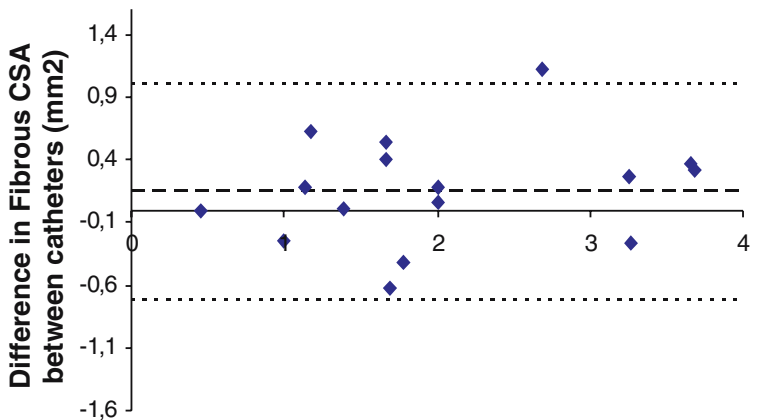

Mean Fibrous CSA (mm2)

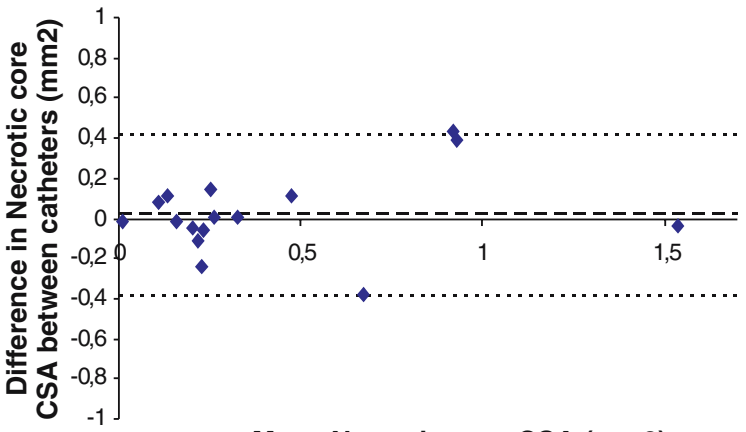

Mean Necrotic core CSA (mm2)

Figure 2. Bland-Altman plots depicting the (observer 1) agreement between catheters for compositional measurements $(n=16)$.

eccentricity $(54 \%)$. The largest relative difference was found in fibrolipidic measurements (Table 4).

Narrow limits of agreement and few outliers (Figures 3 and 4) were found between observers both for geometrical (limits of agreement for lumen, vessel, plaque and plaque burden measurements of $2.61,-2.09 \mathrm{~mm}^{2} ; 2.20-3.03 \mathrm{~mm}^{2} ; 1.70$, $-3.04 \mathrm{~mm}^{2}$; and $9.16,-16.41 \%$; respectively) and compositional (limits of agreement for calcium, fibrous, fibrolipidic and necrotic core measurements of $0.08,-0.09 \mathrm{~mm}^{2} ; 0.89,-1.28 \mathrm{~mm}^{2} ; 0.74$, $-1.06 \mathrm{~mm}^{2}$; and $0.16,-0.20 \mathrm{~mm}^{2}$; respectively) measurements. It is noteworthy that the fibrous and fibrolipidic CSA measurements were highly accurate when assessing cross-sections with small fibrous or fibrolipidic content (Figure 4).

\section{Discussion}

Over the past few years, IVUS has been employed as a tool to assess the temporal effect of conventional and novel drug therapies on coronary plaque size in longitudinal studies $[8,20]$. More recently, the discordance between the beneficial clinical effects of secondary prevention strategies and their effect on plaque volume had lead investigators to explore a potential significant effect on plaque composition [21].

Tissue characterization by means of IVUS radiofrequency (RF) data analysis is a potential tool to enable an accurate evaluation of the composition of coronary plaques [13]. Several investigators have explored the potential of RF data analysis in vivo and reported promising findings [15-17, 22, 23]. This technique has the potential to detect temporal changes in plaque composition and therefore studies have been conducted to assess the effect of conventional drug therapies on the phenotype of coronary atherosclerosis $[18,22,23]$. In addition, there are currently several large trials being conducted with the aim to assess the natural history of high-risk plaques by means of this technique. 
Table 4. Geometrical and compositional measurements of matched CSAs between different observers (n:1316).

\begin{tabular}{|c|c|c|c|c|}
\hline & Observer 1 & Observer 2 & Absolute $\Delta$ & Relative $\Delta(\%)$ \\
\hline \multicolumn{5}{|l|}{ Geometrical data } \\
\hline Lumen CSA $\left(\mathrm{mm}^{2}\right)$ & $10.83 \pm 3.8$ & $10.51 \pm 4.0$ & $0.26 \pm 1.2$ & 2.5 \\
\hline Lumen max. diameter (mm) & $3.96 \pm 0.7$ & $3.86 \pm 0.8$ & $0.08 \pm 0.4$ & 2.1 \\
\hline Lumen min. diameter $(\mathrm{mm})$ & $3.34 \pm 0.6$ & $3.31 \pm 0.7$ & $0.01 \pm 0.3$ & 0.2 \\
\hline Lumen mean diameter (mm) & $3.65 \pm 0.7$ & $3.58 \pm 0.7$ & $0.04 \pm 0.3$ & 1.1 \\
\hline Lumen eccentricity & $0.84 \pm 0.1$ & $0.86 \pm 0.1$ & $0.02 \pm 0.1$ & 2.3 \\
\hline Vessel CSA $\left(\mathrm{mm}^{2}\right)$ & $16.90 \pm 4.2$ & $17.22 \pm 4.3$ & $0.41 \pm 1.3$ & 2.4 \\
\hline Vessel max. diameter (mm) & $4.84 \pm 0.6$ & $4.87 \pm 0.6$ & $0.06 \pm 0.4$ & 1.1 \\
\hline Vessel min. diameter $(\mathrm{mm})$ & $4.30 \pm 0.6$ & $4.35 \pm 0.6$ & $0.08 \pm 0.3$ & 1.7 \\
\hline Vessel mean diameter (mm) & $4.56 \pm 0.6$ & $4.61 \pm 0.6$ & $0.07 \pm 0.3$ & 1.5 \\
\hline Vessel eccentricity & $0.89 \pm 0.1$ & $0.89 \pm 0.1$ & $0.01 \pm 0.1$ & 1.1 \\
\hline Plaque CSA $\left(\mathrm{mm}^{2}\right)$ & $6.07 \pm 2.3$ & $6.72 \pm 2.3$ & $0.67 \pm 1.2$ & 10.3 \\
\hline Plaque max. thickness (mm) & $0.96 \pm 0.3$ & $1.01 \pm 0.3$ & $0.03 \pm 0.2$ & 3.4 \\
\hline Plaque min. thickness (mm) & $0.09 \pm 0.1$ & $0.16 \pm 0.1$ & $0.07 \pm 0.1$ & 53.3 \\
\hline Plaque eccentricity $(\mathrm{mm})$ & $0.09 \pm 0.1$ & $0.16 \pm 0.1$ & $0.07 \pm 0.1$ & 53.6 \\
\hline Plaque burden $(\%)$ & $36.62 \pm 12.2$ & $40.07 \pm 12.5$ & $3.63 \pm 6.4$ & 9.5 \\
\hline \multicolumn{5}{|l|}{ Compositional data } \\
\hline Calcium CSA $\left(\mathrm{mm}^{2}\right)$ & $0.12 \pm 0.2$ & $0.12 \pm 0.2$ & $0.00 \pm 0.0$ & 2.9 \\
\hline Calcium $(\%)$ & $3.66 \pm 6.4$ & $3.51 \pm 6.0$ & $0.13 \pm 2.4$ & 3.7 \\
\hline Fibrous CSA $\left(\mathrm{mm}^{2}\right)$ & $1.86 \pm 1.3$ & $2.05 \pm 1.3$ & $0.20 \pm 0.5$ & 10.2 \\
\hline Fibrous $(\%)$ & $61.48 \pm 18.2$ & $60.05 \pm 18.2$ & $1.10 \pm 14.3$ & 1.8 \\
\hline Fibrolipidic CSA $\left(\mathrm{mm}^{2}\right)$ & $0.60 \pm 0.5$ & $0.75 \pm 0.7$ & $0.16 \pm 0.5$ & 23.5 \\
\hline Fibrolipidic $(\%)$ & $22.34 \pm 14.0$ & $22.09 \pm 15.3$ & $1.86 \pm 11.8$ & 8.8 \\
\hline Necrotic core CSA $\left(\mathrm{mm}^{2}\right)$ & $0.35 \pm 0.4$ & $0.37 \pm 0.4$ & $0.02 \pm 0.1$ & 6.3 \\
\hline Necrotic core $(\%)$ & $10.45 \pm 10.1$ & $10.22 \pm 9.8$ & $0.17 \pm 5.7$ & 1.6 \\
\hline
\end{tabular}

LCSA, VCSA and PCSA refer to lumen, vessel and plaque CSAs. Plaque burden was calculated as $\left[\left(\mathrm{EEM}_{\mathrm{area}}-\mathrm{Lumen}_{\mathrm{area}} / \mathrm{EEM}_{\mathrm{area}}\right) \times\right.$ $100]$.

As the impact of drug therapies on the atherosclerotic plaque burden and composition over time is relatively small, highly reproducible IVUS-VH measurements are essential. Despite such pivotal need for reproducibility data to address the stability of the technique, such studies are lacking. Only indirect evidence of reproducibility has been reported such as a study conducted by our group to assess the 6-month change in plaque composition with no controlled therapeutic intervention [18], and the study conducted by Kawasaki et al. who reported the intra and inter-observer variability of measurements performed using the same pullback [22].

The present study has a unique characteristic since we used two catheters of the same kind at the same time-point, thus simulating the scenario of a longitudinal study.

The main finding of the present study was that IVUS-VH measurements had an acceptable reproducibility. As expected, compositional measurements were more variable than geometrical measurements. Nevertheless, it is noteworthy that inter-catheter differences were predominantly lower than $10 \%$, highly correlated and showed a good agreement. Of note, necrotic core measurements, probably the most relevant component of coronary plaques and currently subject of intense research, showed an excellent inter-catheter and inter-observer agreement. This has a major importance since the temporal change of such component could potentially become an imaging endpoint of longitudinal studies. Similarly, calcium measurements, another important component of atherosclerotic plaques, showed good inter-catheter and inter-observer agreement. The relatively high inter-observer variability of some IVUS-VH variables raises some caution and should be taken into consideration when performing longitudinal studies analyzed by 

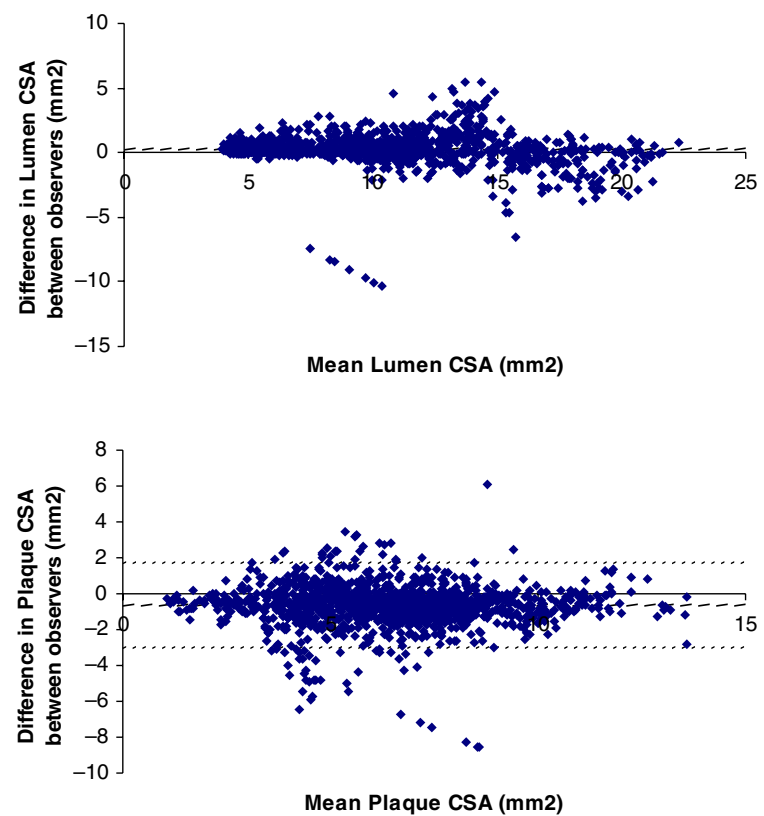

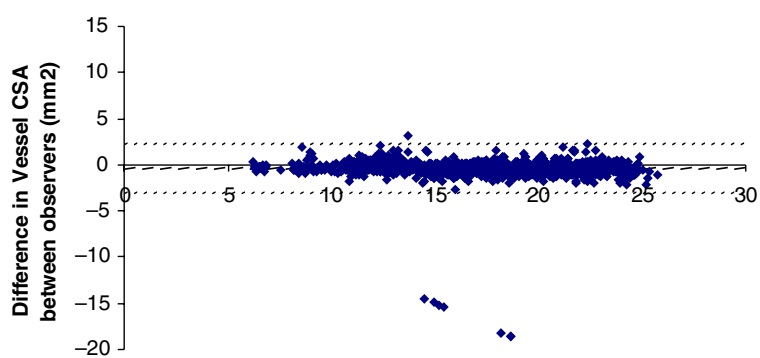

Mean Vesel CSA (mm2)

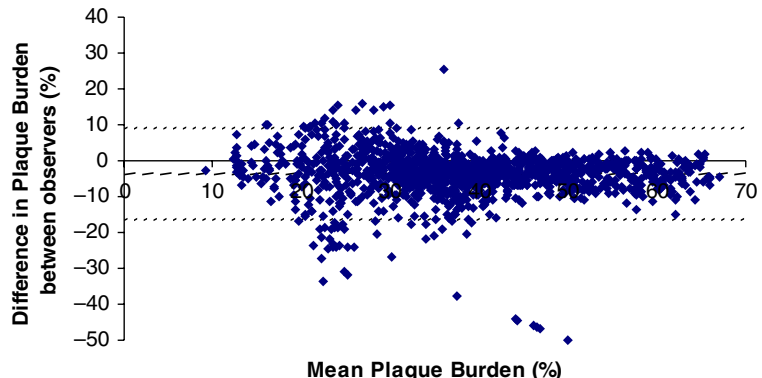

Figure 3. Bland-Altman plots showing the inter-observer agreement for geometrical measurements $(n=1316)$.
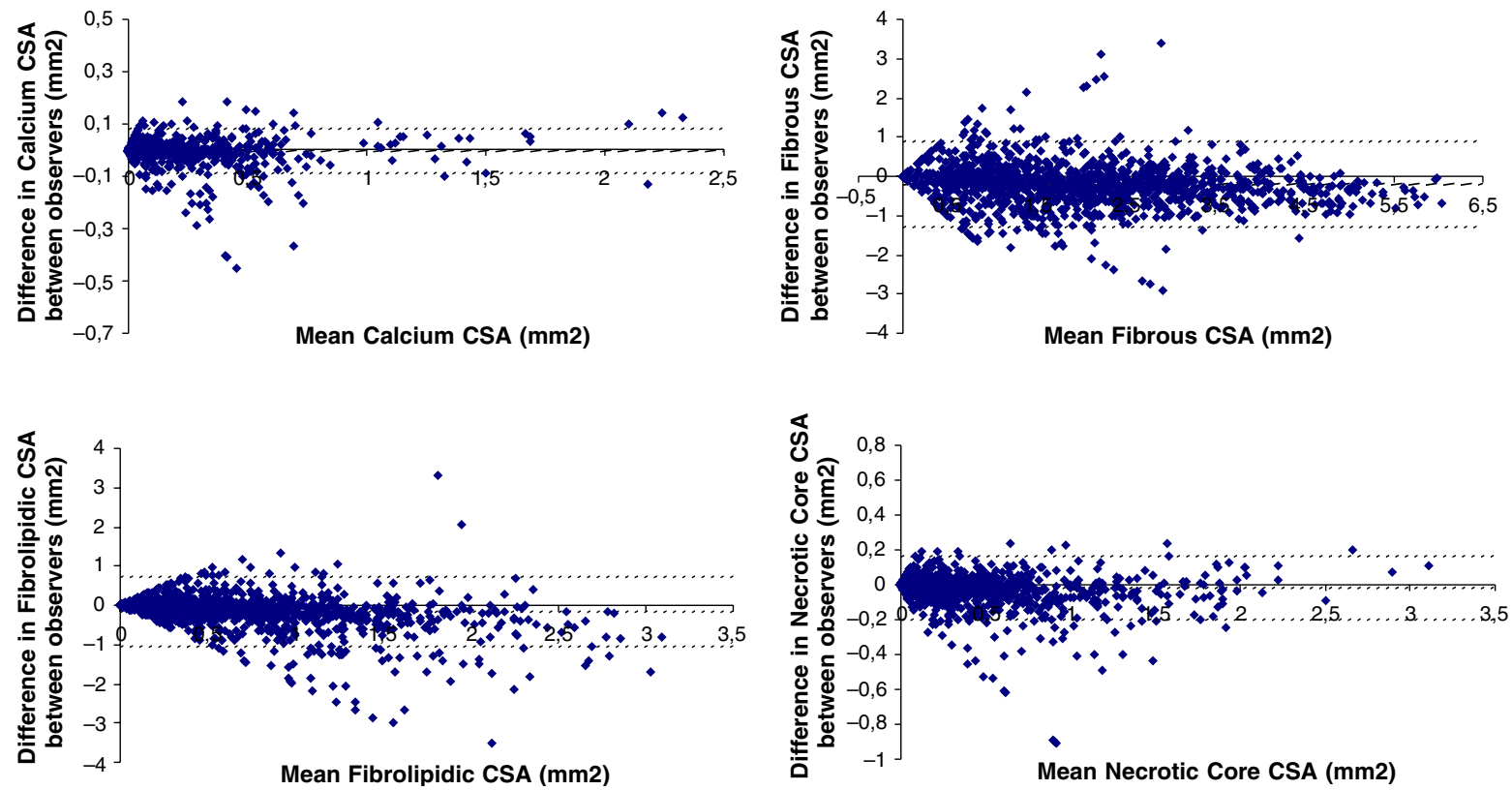

Figure 4. Bland-Altman plots showing the inter-observer agreement for compositional measurements $(n=1316)$. 
core laboratories. Overall, the inter-catheter and inter-observer differences shown might provide boundaries over which changes are statistically significant.

It is evident yet worth mentioning that precise contour detection probably has an essential role in the reproducibility of IVUS-VH measurements. The inter-observer relative difference in plaque CSA measurements was $10 \%$, the commonly accepted threshold. This gives an additive value to our study, since it provides a "real-world" scenario that can aid investigators to perform precise power calculations for longitudinal studies.

Finally, although we aimed at studying nontortuous and non-severely calcified vessels, phased-array IVUS imaging catheters are devoid from a covering sheath and pullbacks are therefore occasionally prone to be non-uniform. This clearly has an impact on determination of the size and composition of atherosclerotic plaques and needs to be taken into consideration for the design of longitudinal studies (Figure 5).

\section{Uniform pullback}
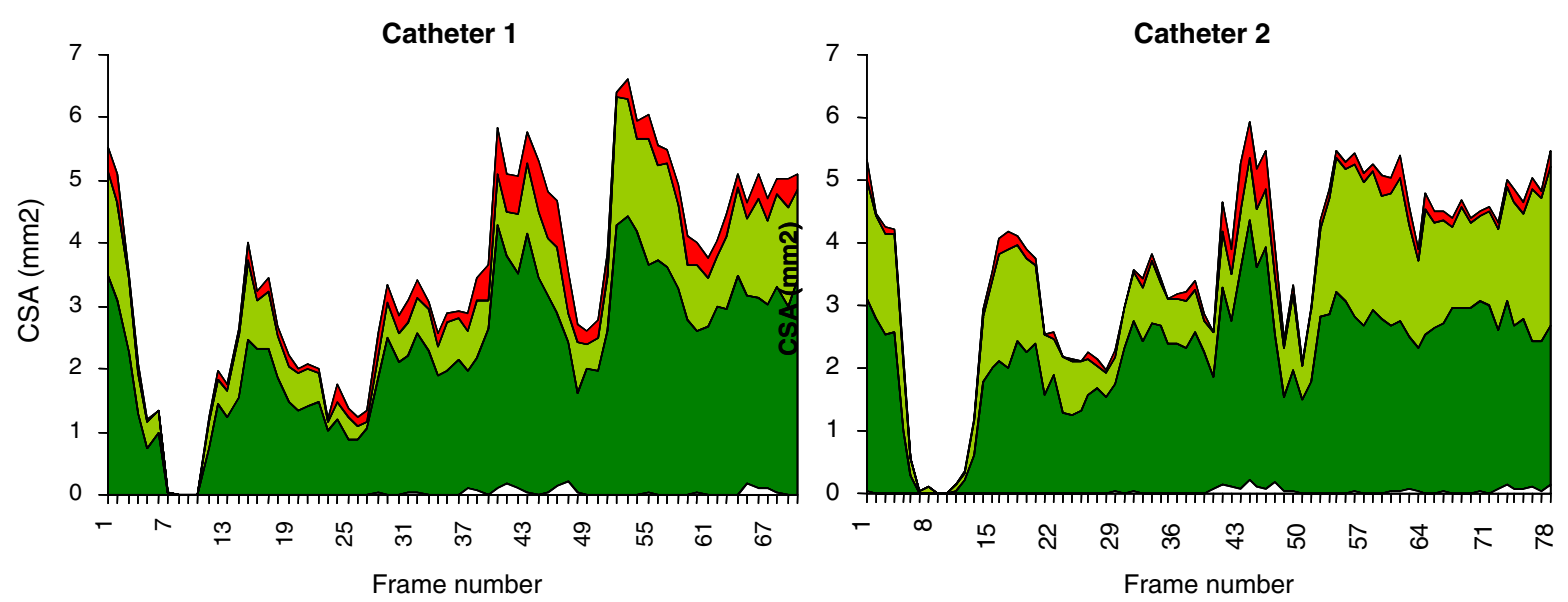

Non-uniform pullback
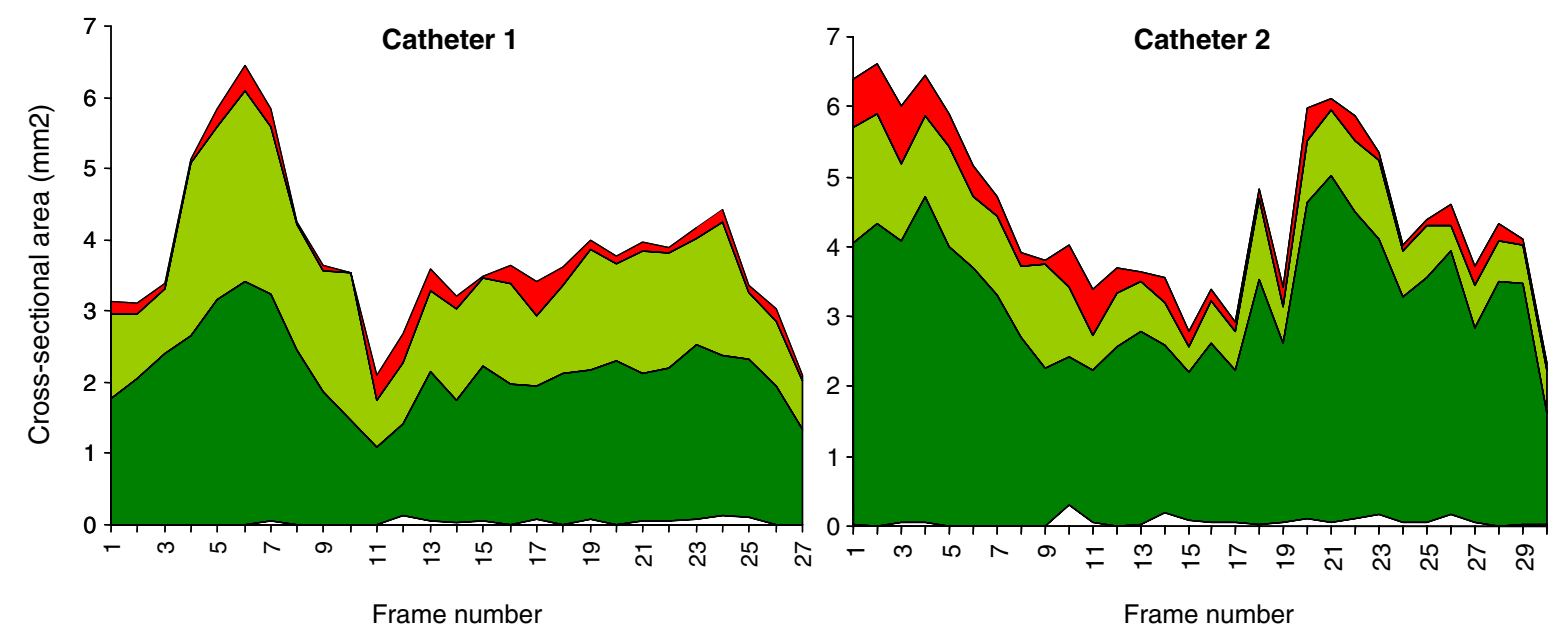

Figure 5. Sequential plotting of a matched ROI interrogated with two catheters. The mean CSA ( $y$ axis) of each plaque component is colour-coded (calcium: white, fibrous: green, fibrolipidic: greenish-yellow and necrotic core: red). This figure shows an example of the impact of non-uniform pullbacks on geometrical and compositional measurements. 


\section{Limitations}

The studied population was relatively small. Nevertheless, the conductance of large in vivo studies of this nature is complicated due to obvious ethical issues. The selection of a population of patients with non-tortuous and non-severely calcified vessels was driven by the aim to study the reproducibility of the technique itself, not of the pullback device. Nevertheless, as shown in Figure 5, the impact of non-uniform pullback speed was not negligible potentially influencing the results.

\section{Conclusions}

The present study demonstrates that the geometrical and compositional output of IVUS-VH is acceptably reproducible. In addition, by providing a "real-world" scenario, this study can aid investigators to perform precise power calculations for longitudinal studies.

\section{References}

1. Guedes A, Keller PF, L'Allier PL, Lesperance J, Gregoire J, Tardif JC. Long-term safety of intravascular ultrasound in nontransplant, nonintervened, atherosclerotic coronary arteries. J Am Coll Cardiol 2005; 45: 559-564.

2. Wenguang L, Gussenhoven WJ, Zhong Y, et al. Validation of quantitative analysis of intravascular ultrasound images. Int J Card Imaging 1991; 6(3-4): 247-253.

3. de Jaegere P, Mudra H, Figulla $\mathrm{H}$, et al. Intravascular ultrasound-guided optimized stent deployment. Immediate and 6 months clinical and angiographic results from the Multicenter Ultrasound Stenting in Coronaries Study (MUSIC Study). Eur Heart J 1998; 19: 1214-1223.

4. Fitzgerald PJ, Oshima A, Hayase M, et al. Final results of the Can Routine Ultrasound Influence Stent Expansion CRUISE study. Circulation 2000; 102: 523-530.

5. Jeremias A, Huegel H, Lee DP, et al. Spatial orientation of atherosclerotic plaque in non-branching coronary artery segments. Atherosclerosis 2000; 152: 209-215.

6. Kimura BJ, Russo RJ, Bhargava V, McDaniel MB, Peterson KL, DeMaria AN. Atheroma morphology and distribution in proximal left anterior descending coronary artery: in vivo observations. J Am Coll Cardiol 1996; 27: 825-831.

7. Smits PC, Pasterkamp G, Quarles van Ufford MA, et al. Coronary artery disease: arterial remodelling and clinical presentation. Heart 1999; 82: 461-464.
8. Nissen SE, Tsunoda T, Tuzcu EM, et al. Effect of recombinant ApoA-I Milano on coronary atherosclerosis in patients with acute coronary syndromes: a randomized controlled trial. JAMA 2003; 290: 2292-2300.

9. Fang JC, Kinlay S, Beltrame J, et al. Effect of vitamins C and $\mathrm{E}$ on progression of transplant-associated arteriosclerosis: a randomised trial. Lancet 2002; 359: 1108-1113.

10. Nissen SE, Tuzcu EM, Schoenhagen P, et al. Effect of intensive compared with moderate lipid-lowering therapy on progression of coronary atherosclerosis: a randomized controlled trial. JAMA 2004; 291: 1071-1080.

11. Davies MJ, Richardson PD, Woolf N, Katz DR, Mann J. Risk of thrombosis in human atherosclerotic plaques: role of extracellular lipid, macrophage, and smooth muscle cell content. Br Heart J 1993; 69: 377-381.

12. Peters RJ, Kok WE, Havenith MG, Rijsterborgh $\mathrm{H}$, van der Wal AC, Visser CA. Histopathologic validation of intracoronary ultrasound imaging. J Am Soc Echocardiogr 1994; 7: 230-241.

13. Nair A, Kuban BD, Tuzcu EM, Schoenhagen P, Nissen SE, Vince DG. Coronary plaque classification with intravascular ultrasound radiofrequency data analysis. Circulation 2002; 106: 2200-2206.

14. Rodriguez-Granillo GA, Aoki J, Ong AT, et al. Methodological considerations and approach to cross-technique comparisons using in vivo coronary plaque characterization based on intravascular ultrasound radiofrequency data analysis: insights from the Integrated Biomarker and Imaging Study (IBIS). Int J Cardiovasc Intervent 2005; 7 : $52-58$.

15. Rodriguez-Granillo GA, Serruys PW, Garcia-Garcia HM, et al. Coronary artery remodelling is related to plaque composition. Heart 2005 Jun 17; (Epub ahead of print).

16. Rodriguez-Granillo GA, Garcia-Garcia H, Mc Fadden E, et al. In vivo intravascular ultrasound-derived thin-cap fibroatheroma detection using ultrasound radiofrequency data analysis. J Am Coll Cardiol 2005; 46(11): 2038-2042.

17. Rodriguez-Granillo GA, McFadden E, Valgimigli M, et al. Coronary plaque composition of non-culprit lesions assessed by in vivo intracoronary ultrasound radio frequency data analysis, is related to clinical presentation. Am Heart $\mathbf{J}$ 2006; In press.

18. Rodriguez-Granillo GA, Serruys PW, Mc Fadden E, et al. First-in-man prospective evaluation of temporal changes in coronary plaque composition by in vivo ultrasound radio frequency data analysis: an integrated biomarker and imaging study (IBIS) substudy. Eurointervention 2005; 3 : 282-288.

19. Bland JM, Altman DG. Statistical methods for assessing agreement between two methods of clinical measurement. Lancet 1986; 1: 307-310.

20. Takagi T, Yoshida K, Akasaka T, Hozumi T, Morioka S, Yoshikawa J. Intravascular ultrasound analysis of reduction in progression of coronary narrowing by treatment with pravastatin. Am J Cardiol 1997; 79: 1673-1676.

21. Schartl M, Bocksch W, Koschyk DH, et al. Use of intravascular ultrasound to compare effects of different strategies 
of lipid-lowering therapy on plaque volume and composition in patients with coronary artery disease. Circulation 2001; 104: 387-392.

22. Kawasaki M, Sano K, Okubo M, et al. Volumetric quantitative analysis of tissue characteristics of coronary plaques after statin therapy using three-dimensional integrated backscatter intravascular ultrasound. J Am Coll Cardiol 2005; 45: 1946-1953.

23. Yokoyama M, Komiyama N, Courtney BK, et al. Plasma low-density lipoprotein reduction and structural effects on coronary atherosclerotic plaques by atorvastatin as clinically assessed with intravascular ultrasound radio-frequency signal analysis: a randomized prospective study. Am Heart $\mathbf{J}$ 2005; 150: 287.

Address for correspondence: Patrick W. Serruys, Thoraxcenter, Bd 406, Dr Molewaterplein 40, 3015 GD, Rotterdam, The Netherlands

Tel.: + 31-10-4635260; Fax: + 31-10-4369154

E-mail: p.w.j.c.serruys@erasmusmc.nl 